\title{
Desarrollo de un sistema electrónico con desplazamiento bidimensional para obtener el flujo lumínico en distintas fuentes de iluminación para conseguir sus curvas características
}

\section{Development of an electronic system with two-dimensional displacement to obtain the luminous flux in different lighting sources to achieve its characteristic curves}

\author{
GONZÁLEZ-GALINDO, Edgar Alfredo†*, VÁZQUEZ-ZAVALA, Laura, SOTO-DELGADO, \\ Douglas Kevin y JIMÉNEZ-QUEZADA, Einar Genaro
}

Universidad Nacional Autónoma de México

ID $1^{\mathrm{er}}$ Autor: Edgar Alfredo, González-Galindo/ ORC ID: 0000-0003-4654-9595, Researcher ID Thomson: G-7927-2018, CVU CONACYT ID: 351785

ID $1^{\text {er }}$ Coautor: Laura, Vázquez-Zavala / ORC ID: 0000-0002-3222-627X, Researcher ID Thomson: C-5585-2019, CVU CONACYT ID: 963138

ID $2^{\text {do }}$ Coautor: Douglas Kevin, Soto-Delgado / ORC ID: 0000-0002-4967-6365, Researcher ID Thomson: C-5598-2019, CVU CONACYT ID: 963141

ID $3^{\text {er }}$ Coautor: Einar Genaro, Jiménez-Quezada / ORC ID: 0000-0003-2282-5794, Researcher ID Thomson: C-83832019, CVU CONACYT ID: 964165

DOI: 10.35429/JEE.2019.9.3.1.9

Recibido: 18 de Abril, 2019; Aceptado 30 de Junio, 2019

\section{Resumen}

Se desarrolló un sistema electrónico controlado con desplazamientos equidistantes de forma perpendicular, para obtener la intensidad lumínica de distintas fuentes de iluminación, con una tarjeta de adquisición de datos para registrar y graficar las curvas características en una cámara obscura. Se implementó una estructura de aluminio colocando bandas dentadas en cada eje y montado sobre el engrane de cada uno de los motores a pasos bipolares, que permiten el movimiento de forma bidimensional. Se generó una interfaz gráfica para controlar la resolución del desplazamiento y se diseñaron soportes en 3D para colocar el sensor de flujo lumínico, obteniendo la curva característica de los distintos tipos de fuentes de iluminación como panel LED, ahorradores, LED de alta potencia e incandescentes en el arreglo experimental. Este tipo de sistema presenta diversos beneficios para aplicaciones en la caracterización de lentes, láseres o bien, en sensores ópticos para aplicarlos en el campo de la electrónica, mecatrónica y robótica. El sistema permite obtener la curva de intensidad lumínica que puede determinar el comportamiento de la fuente luminosa conforme al desplazamiento bidimensional de forma equidistante mostrado en una interfaz gráfica.

Desplazamiento bidimensional, Flujo lumínico, Curvas características

\begin{abstract}
A control electronic system was developed with equidistant perpendicular displacements to obtain the light intensity of different lighting sources, with a data acquisition card to graph the characteristic curves in an obscure chamber. An aluminum structure was implemented, placing bands jagged on each axis and mounted on the gear of each of the with bipolar steps motors, which allow movement in twodimensional form. A graphical interface was generated to control the resolution of the displacement and 3D supports were designed to place the light flow sensor, obtaining the characteristic curve of the different types of lighting sources as LED panel, Compact Fluorescent Lights, high power LED, and incandescent in the experimental arrangement. This type of system presents diverse benefits for applications in the characterization of lenses, lasers or optical sensors to apply them in the field of electronics, mechatronics and robotics. The system allows to obtain the curve of light intensity that can determine the behavior of the light source in accordance with the two-dimensional displacement of equidistant form shown in a graphical interface.
\end{abstract}

Two-dimensional displacement, Luminous flux, Characteristic curves

Citación: GONZÁLEZ-GALINDO, Edgar Alfredo, VÁZQUEZ-ZAVALA, Laura, SOTO-DELGADO, Douglas Kevin y JIMÉNEZQUEZADA, Einar Genaro. Desarrollo de un sistema electrónico con desplazamiento bidimensional para obtener el flujo lumínico en distintas fuentes de iluminación para conseguir sus curvas características. Revista de Ingeniería Eléctrica. 2019 3-9: 1-9

\footnotetext{
* Correspondencia del Autor (unam_alf@comunidad.unam.mx)

$\dagger$ Investigador contribuyendo como primer autor.
} 


\section{Introducción}

En la actualidad la iluminación es sumamente importante en algunas áreas de trabajo, y al mismo tiempo está ligado en el ahorro de energía y a serios problemas de contaminación, para que se conserven tanto el medio ambiente como los recursos, satisfacer las necesidades inmediatas de tareas, sociales, de comportamiento, estéticas, emocionales, de salud y de seguridad. Conocer la intensidad luminosa en distancias pequeñas es sumamente relevante, sabemos que la energía eléctrica se convierte en luz, y la caracterización es mediante varios parámetros, como el flujo luminoso, la luminancia, la iluminación, la distribución de la potencia espectral, la temperatura del color y el índice de reproducción cromática.

Estos parámetros pueden servir también para evaluar la calidad de las fuentes de luz, es un elemento de radiación óptica que reconoce el ojo humano, es decir, en gran medida la longitud de onda visibles que va de los $360 \mathrm{~nm}$ y $830 \mathrm{~nm}$. El enfoque principal son las técnicas de medición, se deben usar dependiendo de las necesidades y tener en cuenta las ventajas como las desventajas de cada una de ellas, conforme a a las necesidades. Uno debe contemplar las características de la fuente de iluminación, pero existe un problema que es vital cuando la fuente de luz esta en movimiento durante el proceso de de evaluación y la recolección de datos. La medición reaccionará muy sensiblemente a un cambio espacial de posición debido a la colocación de la fuente de luz.

En este caso, las únicas técnicas de medición de "distribución de intensidad luminosa" (LID), que pueden considerarse son aquellas que dejan al sujeto en reposo. Las técnicas usadas para medir la intensidad luminosa son: distancia fotométrica de la fuente de luz, ángulo sólido relevante, fuente de luz, contraste relevante, reproducibilidad y exactitud, resolución angular de la LID, tiempo tomado por medidas, requisitos de espacio y por costos que influye la cantidad de espacio debido a la distancia fotométrica (Schwanengel, 2012). En algunas aplicaciones de iluminación, será bueno tener la posibilidad de una medición continua de los parámetros radiométricos, fotométricos $\mathrm{o}$ colorimétricos de las fuentes de luz.
Además, en el proceso para determinar la calidad de las fuentes de iluminación, es importante tener en cuenta el hecho de que los parámetros técnicos de la luz no son constantes en toda la superficie de la fuente. En la Universidad de Tecnología de Bialystok, Polonia trabajan para determinar si es posible medir la distribución de luminancia e iluminancia de la fuente de luz con el apoyo de un robot móvil no demasiado costoso, disponible comercialmente.

Otros estudios que se están haciendo es la medición de luminancia usando una cámara digital equipada con un sensor de imagen para para determinar el resplandor de incomodidad en automóviles los parámetros del dispositivo de medición se basan en número de píxeles, dimensiones del sensor y la distancia o longitud focal, el principal problema detectado fue causado por los diodos emisores de luz en el arreglo de múltiples fuentes de iluminación (Słomiński, 2016). El diseño de investigación deficiente, el tamaño pequeño de las muestras y el uso inadecuado de las pruebas estadísticas, afectan la calidad de la base bibliográfica. Una revisión de la literatura reveló sólo una comprensión débil de las relaciones entre las condiciones luminosas y estas necesidades humanas (Veitch \& Newsham, 1998).

La importancia de algunos trabajos que se están en desarrollo con aplicaciones luminosas es de gran relevancia, la tecnología de neuromodulación precisas en modelos en animales que presentan enfermedades de neuropsiquiatría clínica, estas técnicas están basadas en la exposición de luz usando fibra óptica direccionada a una zona específica del cerebro utilizando como interruptores automáticos, la región del espectro visible de la luz azul $470 \mathrm{~nm}$ y la luz amarilla a $580 \mathrm{~nm}$ (Zhang, Aravanis, Adamantidis, de Lecea, \& Deisseroth, 2007). La importancia de conocer el tipo de fuente de iluminación es de gran importancia para las aplicaciones en los reactores fotoquímicos ya que al exponer un compuesto quimico estos interactúan con los fotones emitidos $\mathrm{y}$ son analizados en los espectrofotómetro de UV-Vis para observar las curvas de Absorbancia y longitud de onda(Montoya Alvarez, 2019; Reinoso Sanchez, 2019). 
La forma de medir la luz en una determianda porción del espectro electromagnetico es mediante un instrumento llamado espectrómetro y esta dentro de la clasificación de los detectores que interactúan con la radiación y la materia y son llamados detectores de centelleo(Herranz Heredia, 2019). Otras de las aplicaciones es la germinación de la semilla ya que es un proceso complejo y sensible, tanto a las condiciones ambientales como en las sustancias endógenas del crecimiento (comúnmente conocidas como fitohormonas).

Dentro de los factores ambientales, se ha observado que las semillas de muchas especies requieren luz para germinar y para algunas, la luz, es un requerimiento obligatorio, en algunas especies el proceso de preparación en las semillas para su germinación tiene hasta un $50 \%$ si está expuesta a la luz infrarroja en el rango del espectro visible que va de $620 \mathrm{~nm}$ a $750 \mathrm{~nm}$ (Fryc \& Dimitrova-Grekow, 2016)

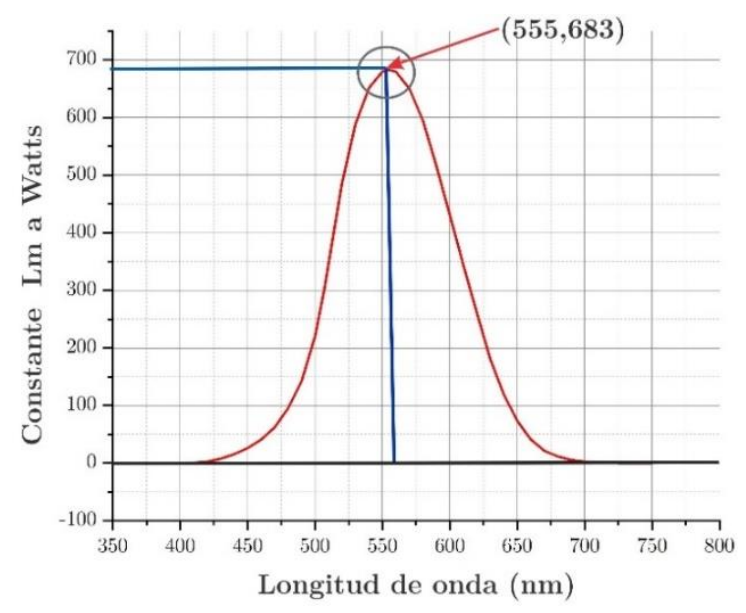

Gráfica 1 Gráfica para obtener las constantes respecto a las longitudes de onda

Para poder realizar la conversión de lúmenes a Watts es necesario tener una constante que permita conocer su equivalencia. En la Gráfica 1 se muestra la coordenada $(555,683)$ donde la longitud de onda es $555 \mathrm{~nm}$ y 683 es la constante 683 lumen $=1$ Watts o 1 lumen $=\mathrm{a}$ 0.001464 Watts. La mayoría de las mediciones radiométricas no requieren un cálculo preciso del área de superficie esférica para convertir entre unidades. Las estimaciones de área plana pueden ser sustituidas por área esférica cuando el ángulo sólido es menor que 0.03 estereorradián, lo que resulta en un error de menos del uno por ciento.
Esto se traduce aproximadamente a una distancia al menos 5 veces mayor que la dimensión más grande del detector. El flujo radiante es una medida de la potencia radiométrica. El flujo, expresado en vatios, es una medida de la tasa de flujo de energía, en Joules por segundo. Dado que la energía fotónica es inversamente proporcional a la longitud de onda, los fotones ultravioletas son más poderosos que los visibles o infrarrojos (Ryer., 1997)

\section{Hipótesis}

Si se tiene un sistema que permita conocer la cantidad de iluminación radiada, por una fuente de luz específica para aplicaciones en distancias pequeñas, entonces se podrá caracterizar las curvas para distintas fuentes de luz emitida y se podrá seleccionar que tipo de fuente y conocer su potencia.

\section{Objetivos}

Desarrollar un sistema con la finalidad de seleccionar una fuente de iluminación para aplicaciones de radiación a pequeñas distancias, es por ello que se realizará un sistema con desplazamiento bidireccional que será controlado por medio de un panel frontal, para la caracterización de la curva de intensidad luminosa.

\section{Metodología}

El trabajo experimental fue desarrollado en el Laboratorio de Medición e Instrumentación y Control, del Centro Tecnológico Aragón (CTA); dicho trabajo es de gran importancia, ya que nos permite seleccionar la fuente de iluminación ideal para una aplicación específica. Se desarrolló una estructura con desplazamiento bidimensional con medidas de $600 \times 400 \mathrm{~mm}$, elaborada con perfil de aluminio estructural 2020 y 2040; para dar movilidad a la estructura, se utilizaron bandas dentadas y motores a pasos bipolares, se diseñaron piezas para dar soporte a un iris al que se le pueden colocar aperturas de $20 \mathrm{~mm} 15 \mathrm{~mm} 10 \mathrm{~mm} 7.5 \mathrm{~mm} 5 \mathrm{~mm}$ y $2.5 \mathrm{~mm}$, se acoplo un luxómetro que presenta las siguientes características; presenta un rangos de 0 a 50,000 luxes $(l x)$ con escalas de $x 1, x 10$ y $x 100 l x$, con una presicion de $+(5 \%)$, dentro de sus especificaciones , trabaja con una temperatura de operacion de $0^{\circ}$ a $40^{\circ} \mathrm{C}$. 


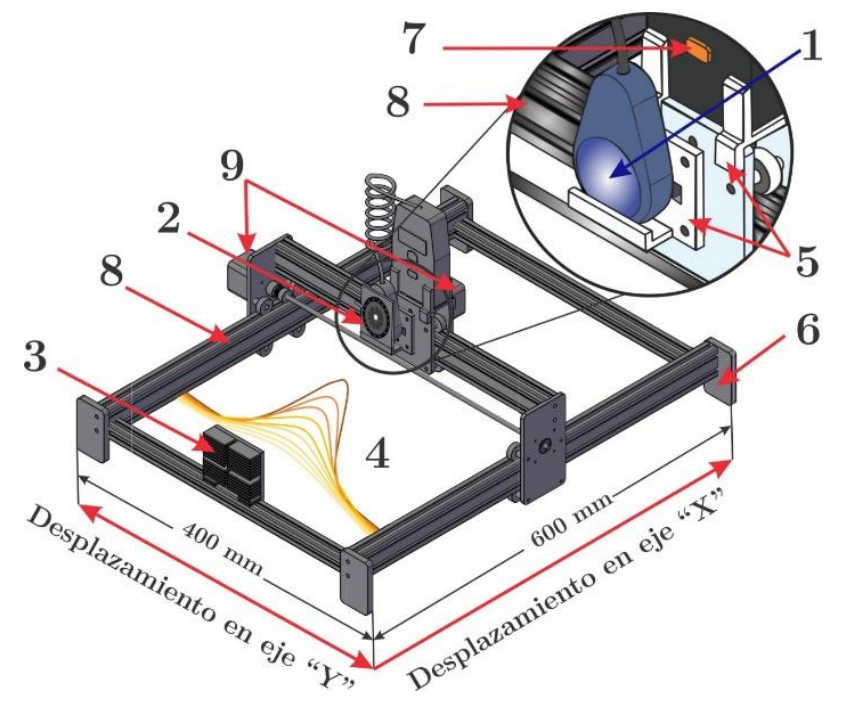

Figura 1 Primer prototipo de un sistema bidireccional para obtener el flujo lumínico

Empleando un software de diseño asistido por computadora del inglés: (ComputerAided Design CAD), se obtuvo el modelo en 3D (E- Alfredo, 2018; Muguruza Blanco, 2019b). El filamento PLA utilizado tiene un diámetro de $1.75 \mathrm{~mm}$ para las piezas impresas. En la estructura del perfil de aluminio se colocó un fotodetector para la adquisición de datos de las diferentes fuentes de iluminación, también se colocó un iris que permite el paso del flujo luminoso que recibe el fotodetector.

En la Figura 1 se representa el sistema bidireccional con el que se hicieron las primeras pruebas para obtener el flujo lumínico, el cual está conformado por los siguientes componentes: 1.-Fotodetector, 2.- Iris (Pinhole), 3.- LED de alta potencia, 4.-Curvas de intensidad, 5.-Pieza impresa en 3D, 6.-Base de acrílico, 7.-Botón Apagado/ Encendido, 8.-Perfil estructurado de aluminio, 9.-Motores a pasos bipolares.

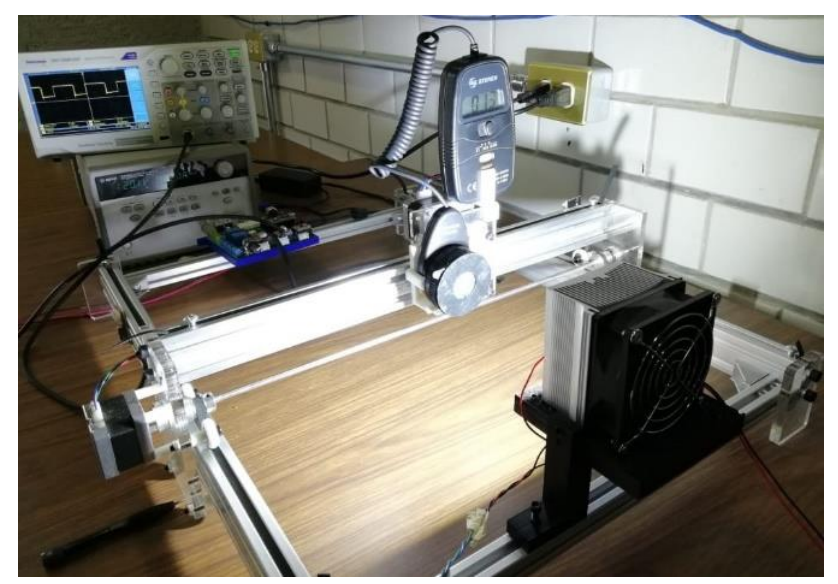

Figura 2 Representación del arreglo experimental de las primeras pruebas con LED de alta potencia (100W) con un fotodetector digital (Luxómetro)

ISSN 2523-2517

ECORFAN® Todos los derechos reservados
En la Figura 2 se puede observar las primeras pruebas del arreglo experimental que se realizó con un fotodetector digital (luxómetro). Antes de comenzar con los experimentos del arreglo final se determinaron puntos de referencia que se usaron para las pruebas en las que se llevaron a cabo barridos del flujo luminoso de distintos tipos de fuentes de iluminación, dentro de las que se encuentran: 1.Foco de 100 Watts de halógeno OSRAM a 125 Volts con una frecuencia de 60 Hertz, de luz cálida con una luminiscencia de $1500 \mathrm{~lm}$ 2.Foco de 72 Watts equivalente a 100 Watts de halógeno ADIR a 120 Volts con una frecuencia de $60 \mathrm{Hertz}$, de luz cálida con una luminiscencia de $1600 \mathrm{~lm}$ 3.-Foco ahorrador de 60 Watts equivalente a 300 Watts ECOSMART a 120 Volts a una frecuencia de $60 \mathrm{Hertz}$, de luz blanca con una luminiscencia $4150 \mathrm{~lm}$ 4.- Foco de 53 Watts de halógeno BrillaMax a 120 volts con una frecuencia de $60 \mathrm{Hertz}$, de luz cálida con una luminiscencia de $1050 \mathrm{~lm} 5$.- Foco de $40 \mathrm{Watts}$ 6.- Led de alta potencia de 100 Watts de 30 volt a $3000 \mathrm{~mA}$ con una luminiscencia de $9000 \mathrm{~lm}$ 7.Led de alta potencia de 33 Watts de 30 volts a $900 \mathrm{~mA}$ con una luminiscencia de $2800 \mathrm{~lm}$ 8.Panel led de alta potencia de 4.4 Watts a 12 Volt.

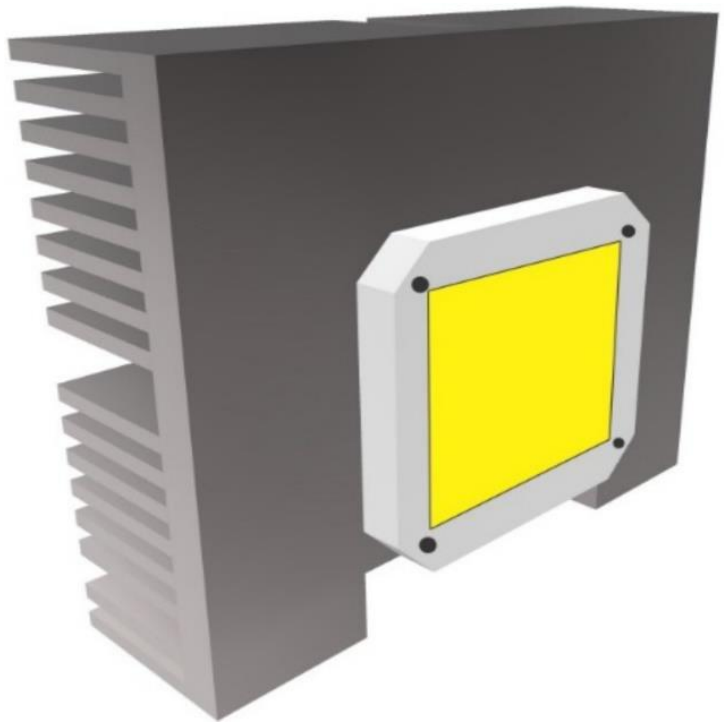

Figura 3 Diodo emisor de luz de alta potencia de $100 \mathrm{~W}$ montado en el sistema

Para las nuevas pruebas de este arreglo experimental, se colocó el fotodetector a una distancia inicial de $10 \mathrm{~mm}$ de separación respecto al eje " $x$ ", se propuso esta distancia ya que si se tomarán mediciones antes de los $10 \mathrm{~mm}$ las lecturas obtenidas no darían como resultado una gráfica tipo gaussiana y no permitiría que fuera útil para algunas aplicaciones.

GONZÁLEZ-GALINDO, Edgar Alfredo, VÁZQUEZ-ZAVALA, Laura, SOTO-DELGADO, Douglas Kevin y JIMÉNEZ-QUEZADA, Einar Genaro. Desarrollo de un sistema electrónico con desplazamiento bidimensional para obtener el flujo lumínico en distintas fuentes de iluminación para conseguir sus curvas características. Revista de Ingeniería Eléctrica. 2019. 
El uso del final de carrera (end stop) como se muestra en la Figura 4 delimita específicamente el punto de origen y término para desplazarse sobre el eje " $y$ ", se presenta, la misma condición de desplazamiento para el eje " $x$ " a lo largo del perfil de aluminio 2040, con una separación de $260 \mathrm{~mm}$ entre ellos. El punto de partida de nuestro sistema será determinado en la coordenada $(x, y)$ donde $x=10 \mathrm{~mm}$ y $y=0$ $\mathrm{mm}$. Para la captura de los datos se realizó un desplazamiento sobre el eje " $y$ ", con las coordenadas $(x, y)$, donde $x=10 \mathrm{~mm}$ y $y=10$ $\mathrm{mm}, 20 \mathrm{~mm}, 30 \mathrm{~mm}, \ldots, 260 \mathrm{~mm}$; cuando $y=$ $260 \mathrm{~mm}$, esta regresara a su coordenada $y=0 \mathrm{~mm}$ finalizando así el primer recorrido, para el segundo recorrido, se realiza un desplazamiento de $10 \mathrm{~mm}$ sobre el eje $x$ dando la coordenada ( $x$, y) $\operatorname{con} x=20 \mathrm{~mm}$ y $y=10 \mathrm{~mm}, 20 \mathrm{~mm}, 30 \mathrm{~mm}$, ... , $260 \mathrm{~mm}$, finalizando así el segundo recorrido. Repitiendo este procedimiento en los siguientes recorridos hasta llegar a la coordenada $(110 \mathrm{~mm}, 260 \mathrm{~mm})$.

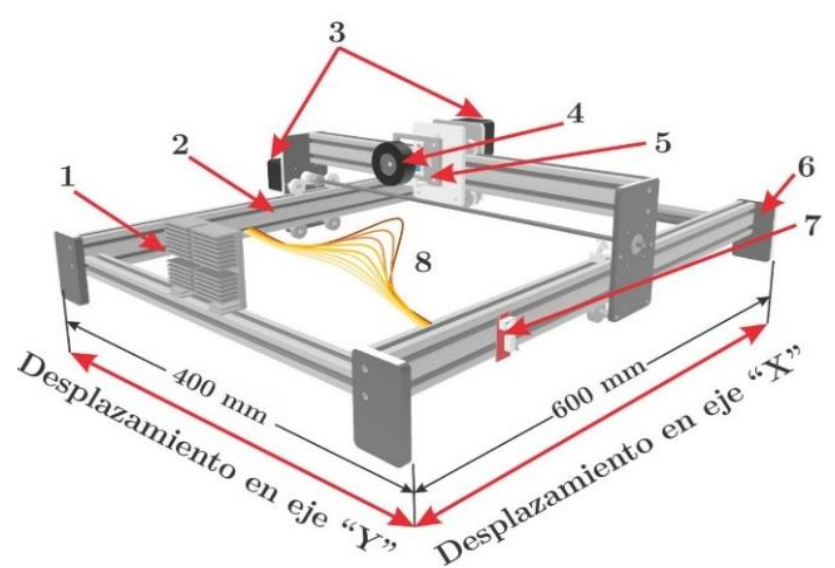

Figura 4 Representación del nuevo arreglo experimental en pruebas con LED de alta potencia (100W)

En la Figura 4 se representa el nuevo sistema bidireccional para obtener el flujo lumínico, el cual se describe de la siguiente manera: 1.-LED de alta potencia, 2.-Perfil estructurado de aluminio, 3.-Motores a pasos bipolares, 4.-Iris (Pinhole) 5.- Sensor de luz modelo $M D 0338$ en el rango de la luz visible de $560 \mathrm{mn}$, con un rango de entrada de 1-65535 lx, 6.-Base de acrílico, 7.-Final de carrera (end stop) y 8.-Curvas de intensidad. Para realizar el movimiento bidimensional de nuestro sistema se llevó a cabo el diseño de una placa PCB (por sus siglas en inglés Printed Circuit Board), en la Figura 5 se muestra la tarjeta de propósito general con los componentes electrónicos que se emplearon para el desarrollo de esta y en la Figura 6 se observa el circuito esquemático de la tarjeta de circuito impreso.

ISSN 2523-2517

ECORFAN $®$ Todos los derechos reservados

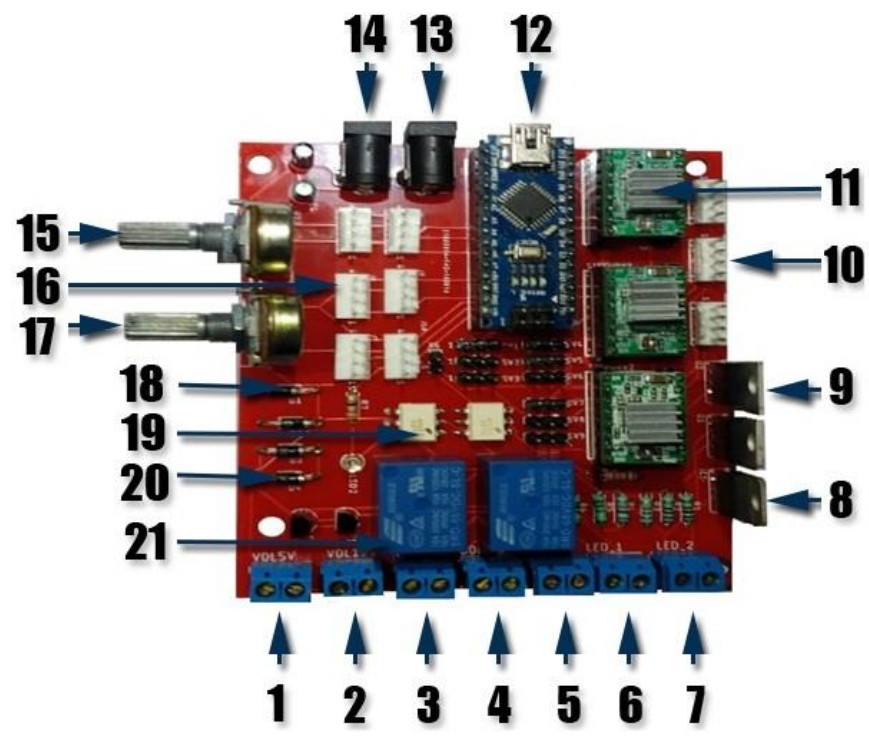

Figura 5 Tarjeta de control de propósito general empleada en el arreglo experimental

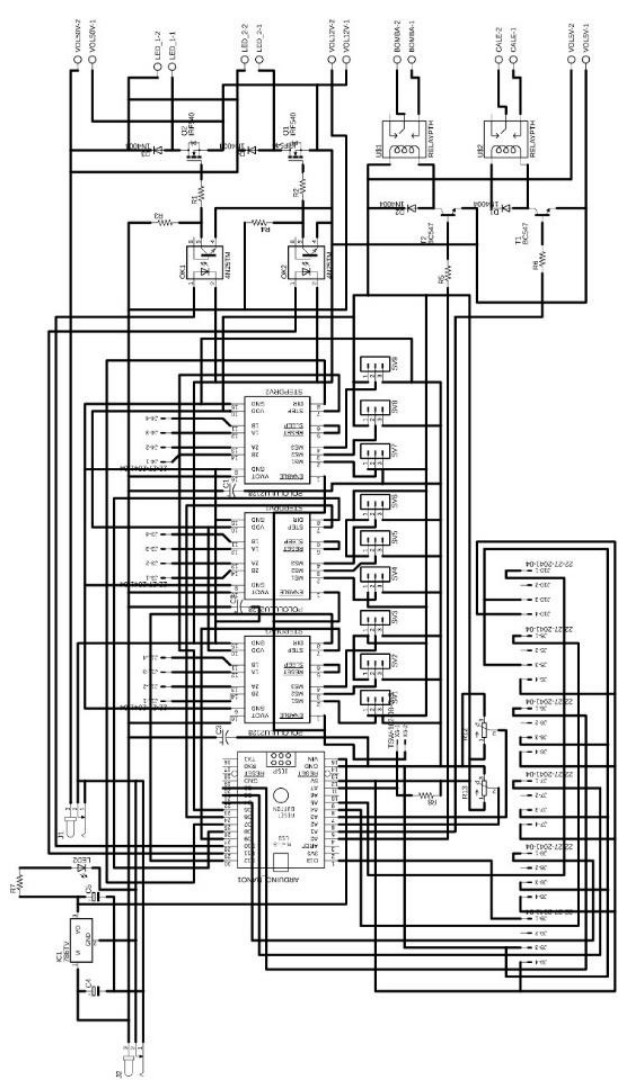

Figura 6 Circuito esquemático correspondiente a la placa de propósito general usado en el sistema

La descripción de la tarjeta de control de propósito general se explica de la siguiente manera: 1.-Bornera para entrada de 5 volts, 2.Bornera para entrada de 12 volts, 3.- Bornera para entrada de 50 volts, 4.- Bornera para salida de Bomba, 5.- Bornera para salida de Calentador, 6.- Bornera para salida de LED 1, 7.Bornera para salida de LED 2, 8.- Transistor regulador de voltaje $L M 7805$, 9.- Transistor MOSFET IR3205, 10.- Pin Header para la conexión de los motores bipolares.

GONZÁLEZ-GALINDO, Edgar Alfredo, VÁZQUEZ-ZAVALA, Laura, SOTO-DELGADO, Douglas Kevin y JIMÉNEZ-QUEZADA, Einar Genaro. Desarrollo de un sistema electrónico con desplazamiento bidimensional para obtener el flujo lumínico en distintas fuentes de iluminación para conseguir sus curvas características. Revista de Ingeniería Eléctrica. 2019. 
11.- Módulo controlador de motores A4988, 12.- Tarjeta de desarrollo Arduino NANO, 13.- Conector Jack hembra para alimentación de 50 volts, 14.- Conector Jack hembra para alimentación de 12 volts, 15.Potenciómetro para la regulación de la intensidad luminosa en el LED 1, 16.- Pin Header para la conexión de los finales de carrera, 17.- Potenciómetro para la regulación de la intensidad luminosa en el LED 2, 18.- Diodos rectificadores para protección de los transistores IR3205, 19.- Optoacoplador 4N27, 20.- Diodos rectificadores para protección de los Relevadores de 5 Volts y 21.- Relevadores de 5 Volts.

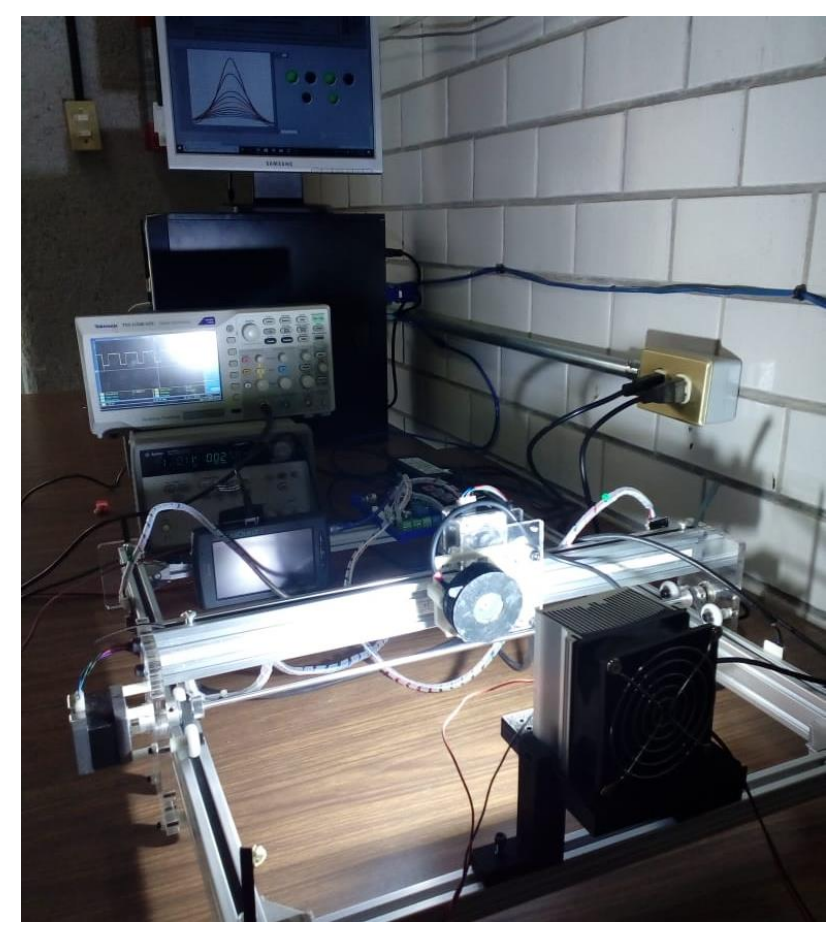

Figura 7 Arreglo experimental del sistema bidireccional para obtener el flujo lumínico con un diodo emisor de luz (LED) de alta potencia de 100 Watts

La Figura 7 muestra el sistema bidireccional usado para obtener el flujo lumínico de las fuentes de iluminación, es importante mencionar que los motores bipolares implementados en el sistema tienen una resolución de desplazamiento de aproximadamente $200 \mu \mathrm{m}$ por paso; a pesar de que existen motores a pasos que presentan una mejor resolución como podrían ser los motores a pasos inteligentes con una resolución de hasta 2 $\mu m$, su costo es elevado(Muguruza Blanco, 2019a).
Para la obtención de los datos se empleó una interfaz gráfica, también se utilizó un osciloscopio con el cual se observa el PWM (por sus siglas en inglés Pulse-Width Modulation), proporcionado por la tarjeta de control de propósito general, con la cual se realizó la adquisición de los datos de las fuentes lumínicas.

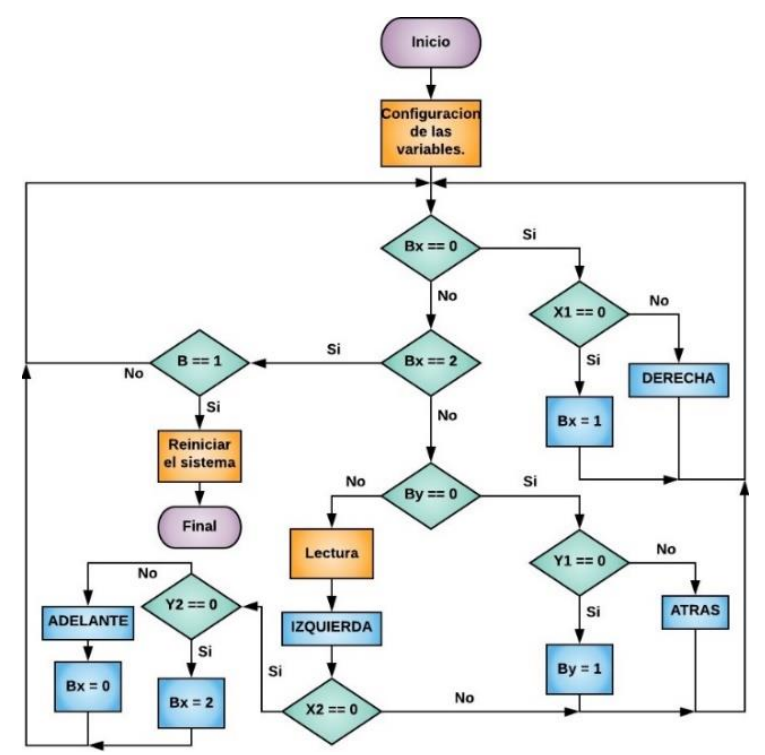

Figura 8 Diagrama de flujo empleado para el código de la tarjeta de control para su funcionamiento

El diagrama de flujo empleado para el código de control del desplazamiento del sistema bidireccional (Figura 8) presenta las siguientes variables: $B x=$ Bandera para el eje $X, B y=$ Bandera para el eje $Y, X 1=$ Sensor final de carrera en $X$ posición inicial, $X 2=$ Sensor final de carrera en $X$ posición final, $Y 1=$ Sensor final de carrera en $Y$ posición inicial, $Y 2=$ Sensor final de carrera en $Y$ posición final, $B=$ Botón para el reinicio, DERECHA = Desplazamiento del Luxómetro de 260mm en $X+1$, IZQUIERDA = Desplazamiento del Luxómetro de $10 \mathrm{~mm}$ en $X$ 1, ADELANTE = Desplazamiento del Luxómetro de $10 m m$ en $Y+1, A T R A ́ S=$ Desplazamiento del Luxómetro de 320mm en $Y-1$, Lectura = Adquisición de datos con el Luxómetro.

\section{Resultados}

Con las lecturas conseguidas del arreglo experimental de las distintas fuentes de iluminación, se realizó una comparación de los valores máximos de iluminación, como se muestra en la Tabla 1. 


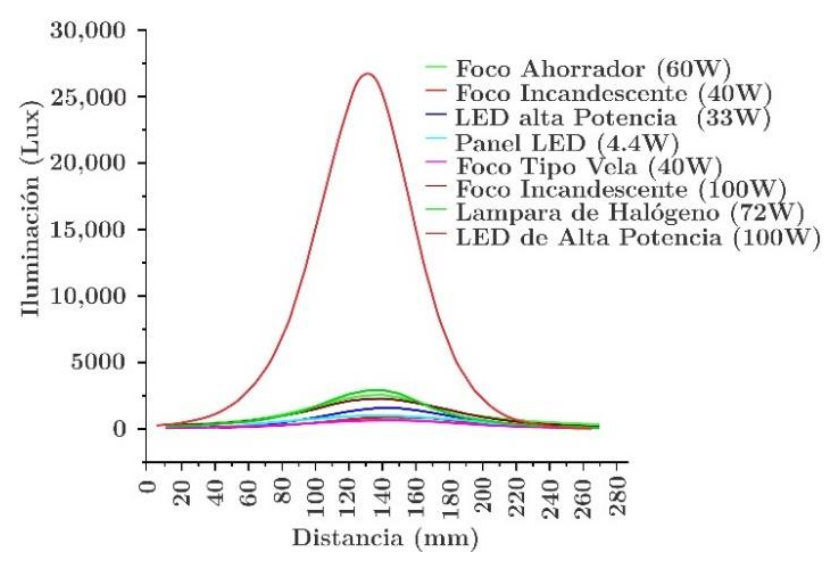

Gráfica 2 Comparativa de todas las fuentes de iluminación empleadas en el arreglo experimental

En la Gráfica 2 se aprecia el comportamiento de la intensidad luminosa obtenida a través la adquisición de datos y representado gráficamente los puntos máximos de cada fuente usadas en el arreglo experimental como se muestra en la Figura 7 usando un LED de alta potencia de 100 Watts dando un punto máximo de 27,100 luxes o su equivalente en potencia de $39.677 \frac{\mathrm{W}}{\mathrm{m}^{2}}$. Se realizó una comparación entre dos fuentes de iluminación, ya que con ellas se obtuvieron los mejores resultados del arreglo experimental; en la Gráfica 3 se puede observar que existe una diferencia de $\approx 25,000$ luxes entre sus puntos máximos.

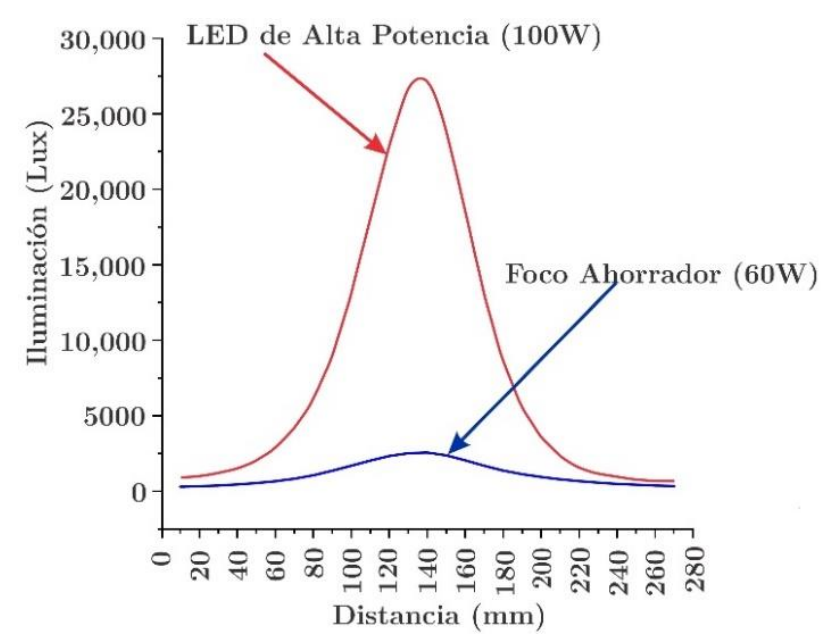

Gráfica 3 Gráfica comparativa de las fuentes de iluminación Foco ahorrador de 60W equivalente a 300 Watts y LED de alta potencia de $100 \mathrm{~W}$

La Gráfica 4 muestra el barrido de todos los datos obtenidos por el arreglo para el diodo emisor de luz (LED) de alta potencia a 100W.
A lo largo del barrido se puede observar que conforme se aumenta la distancia de separación entre el fotodetector y la fuente de iluminación en el eje " $x$ " el valor de los luxes en el punto máximo se disminuyen de forma muy significativa, mientras que a los extremos del eje " $y$ " se obtienen valores muy similares entre sí, sin importar la distancia que se tenga en el eje " $x$ ".

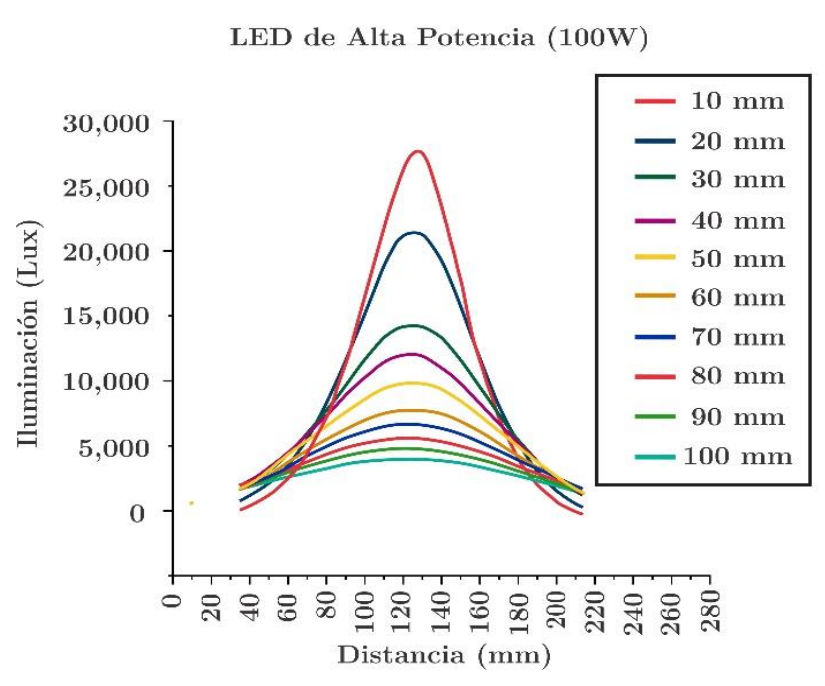

Gráfica 4 Gráfica de barridos del LED de alta potencia $(100 \mathrm{~W})$

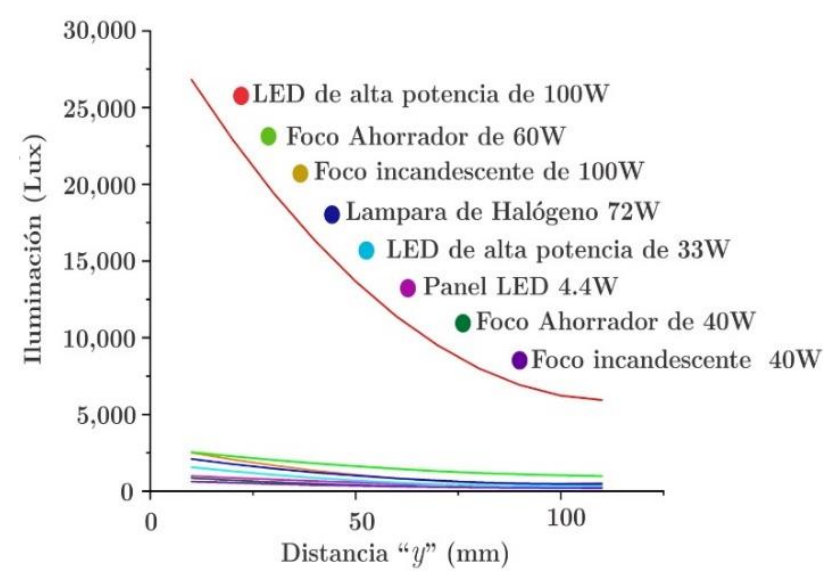

Gráfica 5 Gráfica comparativa de puntos máximos con unidades de iluminación (Lux)

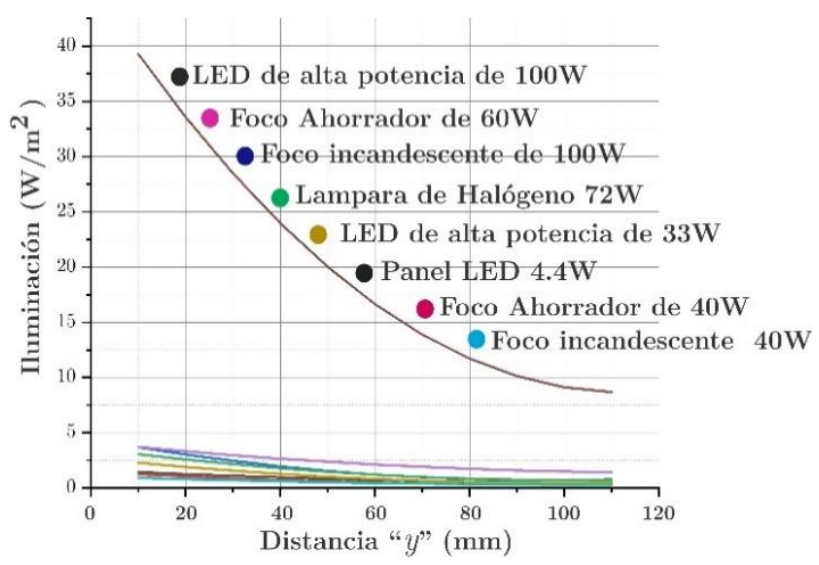

Gráfica 6 Gráfica comparativa de puntos máximos con unidades de potencia $\frac{\mathrm{W}}{\mathrm{m}^{2}}$

GONZÁLEZ-GALINDO, Edgar Alfredo, VÁZQUEZ-ZAVALA, Laura, SOTO-DELGADO, Douglas Kevin y JIMÉNEZ-QUEZADA, Einar Genaro. Desarrollo de un sistema electrónico con desplazamiento bidimensional para obtener el flujo lumínico en distintas fuentes de iluminación para conseguir sus curvas características. Revista de Ingeniería Eléctrica. 2019. 
En las gráficas 5 y 6 se observa una comparación de los puntos máximos obtenidos por cada una de las fuentes de iluminación, donde el eje de las abscisas representa la distancia en $\mathrm{mm}$ y el eje de las ordenadas representa la intensidad luminosa en las unidades de luxes para la Gráfica 5 y su conversión a $\frac{W}{m^{2}}$ para la Gráfica 6; en ambas gráficas se puede observar que el LED de alta potencia de 100W muestra los mejores resultados ya que presenta una diferencia de 24,430 luxes o $35.7684516 \mathrm{Wm} 2$ con referencia a la segunda fuente con mejores resultados (Lámpara de halógeno 72W).

De la Tabla 1. podemos observar que el diodo emisor de luz (LED) de alta potencia a 100W tiene una mayor intensidad luminosa, ya que alcanza los 27,100 luxes cuando se encuentra posicionado en la coordenada $(10 \mathrm{~mm}$, $130 \mathrm{~mm}$ ) del sistema empleado, como se muestra en la Gráfica 2.

\begin{tabular}{|c|c|c|c|c|c|c|c|}
\hline 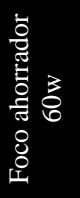 & 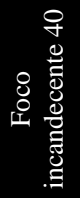 & 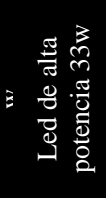 & 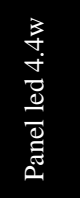 & 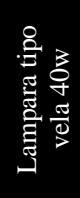 & 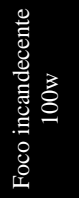 & 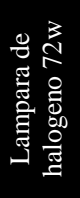 & 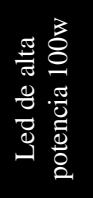 \\
\hline 340 & 98 & 90 & 50 & 61 & 170 & 100 & 600 \\
\hline 380 & 119 & 110 & 70 & 75 & 210 & 130 & 600 \\
\hline 430 & 146 & 150 & 90 & \begin{tabular}{|l|}
93 \\
\end{tabular} & 270 & 160 & 700 \\
\hline 490 & 179 & 190 & 110 & 116 & 340 & 200 & 900 \\
\hline 570 & 222 & 250 & 130 & 145 & 420 & 270 & 1100 \\
\hline 670 & 278 & 340 & 190 & 186 & 540 & 360 & 1500 \\
\hline 790 & 348 & 450 & 340 & 237 & 700 & 470 & 2300 \\
\hline 950 & 433 & 590 & 510 & 303 & 890 & 650 & 3600 \\
\hline 1130 & 531 & 770 & 630 & 382 & 1130 & 850 & 5600 \\
\hline 1370 & 662 & 980 & 730 & 467 & 1430 & 1150 & 8800 \\
\hline 1690 & 797 & 1210 & 830 & 548 & 1700 & 1560 & 13300 \\
\hline 2050 & 856 & 1420 & 910 & 612 & 1960 & 2120 & 18900 \\
\hline 2380 & 870 & 1560 & 970 & \begin{tabular}{|l|}
644 \\
\end{tabular} & 2180 & 2670 & 24200 \\
\hline 2560 & 875 & 1610 & 1000 & 647 & 2290 & 2930 & 27100 \\
\hline 2520 & 816 & 1490 & 990 & 625 & 2250 & 2880 & 26400 \\
\hline 2330 & 718 & 1320 & 940 & 587 & 2110 & 2620 & 22500 \\
\hline 2030 & 592 & 1090 & 860 & 525 & 1850 & 2180 & 17600 \\
\hline 1690 & 468 & 860 & 770 & 447 & 1500 & 1680 & 12700 \\
\hline 1360 & 360 & 650 & 660 & 368 & 1210 & 1260 & 8600 \\
\hline 1060 & 274 & 490 & 550 & 305 & 970 & 980 & 5800 \\
\hline 830 & 209 & 350 & 440 & 247 & 750 & 730 & 4000 \\
\hline 660 & 160 & 260 & 320 & 201 & 590 & 550 & 2700 \\
\hline 540 & 123 & 190 & 180 & 163 & 480 & 450 & 1900 \\
\hline 450 & 97 & 140 & 110 & 133 & 390 & 350 & 1400 \\
\hline 380 & 78 & 110 & 80 & 110 & 330 & 270 & 1100 \\
\hline 330 & 63 & 80 & 60 & 92 & 290 & 220 & 900 \\
\hline 290 & 52 & 70 & 50 & 76 & 230 & 180 & 800 \\
\hline
\end{tabular}

Tabla 1 Comparación de las distintas fuentes de iluminación usados en el arreglo experimental
La Tabla 2 presenta los datos de los puntos máximos en unidades de potencia $\mathrm{Wm} 2$ de las distintas fuentes de iluminación, en dicha tabla se observa que el diodo emisor de luz (LED) de alta potencia a $100 \mathrm{~W}$ continúa presentando los valores más altos, alcanzando un valor de $\approx 40 \frac{\mathrm{W}}{\mathrm{m}^{2}}$ cuando se encuentra posicionado en la coordenada $(10 \mathrm{~mm}, 130 \mathrm{~mm})$ del sistema empleado.

\begin{tabular}{|c|c|c|c|c|c|c|c|}
\hline 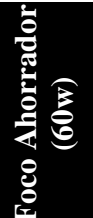 & 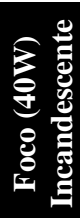 & 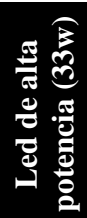 & 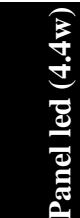 & 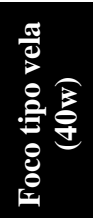 & 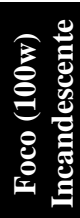 & 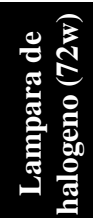 & 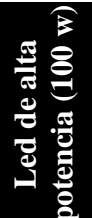 \\
\hline 48 & 281 & 357 & .464 & 0.943 & .192 & ) & \\
\hline 294 & 025 & 39 & 1.259 & 0.801 & 548 & 2.958 & .67 \\
\hline 2.987 & 836 & 508 & 1.098 & 0.678 & 2.064 & .2 & \\
\hline 2.635 & 0.695 & 230 & 0.937 & 0.589 & 1.713 & 1.8 & \\
\hline 357 & 0.586 & 1 & 0.8 & 0.508 & 1.449 & 1.5 & \\
\hline 123 & 02 & & 0 & 0.4 & 1.230 & 1.2 & \\
\hline 947 & 35 & & & 0.394 & 1. & & \\
\hline 772 & 78 & & & 0.357 & 0.922 & 0.92 & \\
\hline 511 & 2 & 42 & 7 & 0.316 & .791 & 0.80 & \\
\hline 77 & & & 4 & 0.284 & 703 & \begin{tabular}{|l|}
0.7 \\
\end{tabular} & \\
\hline 1.420 & 64 & 0.425 & 0.395 & 0.255 & 0.615 & 0.630 & \\
\hline
\end{tabular}

Tabla 2 Comparación de puntos máximos y su conversión de Lux a W/m2 de las distintas fuentes de iluminación usadas en el arreglo experimental

\section{Conclusiones}

En la actualidad existen sistemas que controlan la intensidad de iluminación que son simples y fáciles, que cubren los metros cuadrados regidos por la NORMA Oficial Mexicana NOM-025STPS-2008 para los niveles mínimos de iluminación que deben incidir en el plano de trabajo. Algunos sistemas tienen fuentes de iluminación adaptados para trabajar en áreas pequeñas, pero se desconoce la intensidad luminosa que se emite de la fuente al área de trabajo, como son los microscopios, las lupas, las estaciones de trabajo para soldar, los reactores fotoquímicos etc.

El sistema desarrollado que realiza el desplazamiento bidireccional para la caracterización de la curva de intensidad luminosa permite saber cuál es la potencia emitida a una distancia pequeña de distintas fuentes de iluminación si comparamos los datos adquiridos de dos fuentes de iluminación como se muestra en la Tabla 2 de un foco incandescente de 100 Watts y un diodo emisor de luz (LED) de 100 Watts, se observó que es de aproximadamente 12.5 veces mayor la potencia de un diodo emisor de Luz de 100 Watts.

GONZÁLEZ-GALINDO, Edgar Alfredo, VÁZQUEZ-ZAVALA, Laura, SOTO-DELGADO, Douglas Kevin y JIMÉNEZ-QUEZADA, Einar Genaro. Desarrollo de un sistema electrónico con desplazamiento bidimensional para obtener el flujo lumínico en distintas fuentes de iluminación para conseguir sus curvas características. Revista de Ingeniería Eléctrica. 2019. 
El arreglo experimental permite la irradiación controlada de luz a diferentes distancias (24,540 luxes), cubriendo un amplio rango de aplicación y de esta manera cubrir las necesidades de cada diseñador.

\section{Agradecimiento}

Los autores agradecen al Centro Tecnológico de la Facultad de Estudios Superiores Aragón de la Universidad Nacional Autónoma de México, por las facilidades en el uso del Laboratorio de Medición e Instrumentación y Control, al Laboratorio de Ingeniería Ambiental y al Programa de Apoyo a Proyectos para la Innovación y Mejoramiento de la Enseñanza (PAPIME) con No. de clave PE105519, también se agradece a Omar Yair Espinosa Luna y Oscar Abraham Luy Marín por su colaboración técnica.

\section{Referencias}

E- Alfredo, G.-G. (2018). Diseño de una superficie de revolución libre de forma impreso en 3D como concentrador solar tipo Fresnel. Congreso Internacional de Investigación e Innovación 2018, 3, 8377-8388.

Fryc, I., \& Dimitrova-Grekow, T. (2016, 13-16 Sept. 2016). An automated system for evaluation of the quality of light sources. Paper presented at the 2016 IEEE Lighting Conference of the Visegrad Countries (Lumen V4).

Herranz Heredia, E. (2019). Estudio de técnicas de imagen, radiaciones ionizantes y sus aplicaciones en radioterapia. 35-36.

Montoya Alvarez, X. J. (2019). Informe Tecnico Diseño de colores en Fibra de Alpaca en un Laboratorio de una Empresa Textil. 39-40.

Muguruza Blanco, A. (2019a). Contribuci na las tecnolog as de fabricaci $n$ aditiva para la obtenci $\mathrm{n}$ de piezas multimaterial, combinando la impresi $\mathrm{n}$ 3D por $\mathrm{m}$ scara con la impresi $\mathrm{n}$ funcional mediante sistemas InkJet (Vol. 2).

Muguruza Blanco, A. (2019b). Contribución a las tecnologías de fabricación aditiva para la obtención de piezas multimaterial, combinando la impresión 3D por máscara con la impresión funcional mediante sistemas InkJet (Vol. 1).
Reinoso Sanchez, M. A. (2019). Fabricación de celdas híbridas con arquitectura planar basadas en compuestos metal-orgánicos con estructura perovskita. Universidad Nacional de ColombiaSede Bogotá, Retrieved from http://repositorio.unsa.edu.pe/bitstream/handle/ UNSA/8691/IQmoalxj.pdf?sequence=1\&isAllo wed $=\mathrm{y}$

Ryer., A. (1997). The Light Measurement Han. International Light Technologies, 1, 30-40.

Schwanengel, C. J. Ç. A. h. w. t. d. r. d. L. M. p. (2012). Comparison of techniques for measuring luminous intensity distribution overall and across segments.

Słomiński, S. (2016). Identifying problems with luminaire luminance measurements for discomfort glare analysis. Lighting Research \& Technology, 48(5), 573-588. doi:10.1177/1477153515596374

Veitch, J. A., \& Newsham, G. R. J. J. o. t. I. E. S. (1998). Lighting quality and energyefficiency effects on task performance, mood, health, satisfaction, and comfort. 27(1), 107-129.

Zhang, F., Aravanis, A. M., Adamantidis, A., de Lecea, L., \& Deisseroth, K. (2007). Circuitbreakers: optical technologies for probing neural signals and systems. Nature Reviews Neuroscience, 8, 577. doi:10.1038/nrn2192 